\title{
Analysis of Vibroacoustic Modulations for Crack Detection: A Time-Frequency Approach Based on Zhao-Atlas-Marks Distribution
}

\author{
A. Trochidis, ${ }^{1}$ L. Hadjileontiadis, ${ }^{2}$ and K. Zacharias ${ }^{1}$ \\ ${ }^{1}$ Department of Mathematical, Physical and Computational Sciences, Aristotle University of Thessaloniki, 54124 Thessaloniki, Greece \\ ${ }^{2}$ Department of Electrical \& Computer Engineering, Aristotle University of Thessaloniki, University Campus, 54124 Thessaloniki, Greece
}

Correspondence should be addressed to L. Hadjileontiadis; leontios@auth.gr

Received 11 July 2013; Accepted 7 January 2014; Published 12 June 2014

Academic Editor: Nuno Maia

Copyright (C) 2014 A. Trochidis et al. This is an open access article distributed under the Creative Commons Attribution License, which permits unrestricted use, distribution, and reproduction in any medium, provided the original work is properly cited.

\begin{abstract}
The vibro-acoustic modulation (VAM) technique is probably the most widely used nonlinear method for crack detection. The VAM method is based on the effect of modulation of high-frequency acoustic waves by a low-frequency vibration. The intensity of the modulation is related to the severity of the damage and has been used so far as a damage index. The damage index simply based on the amplitude of the first side bands in the spectral domain often leads to controversial results about the severity of the damage. In this work, the nonlinear characteristics of the vibro-modulation were systematically investigated by employing time-frequency analysis based on the Zhao-Atlas-Marks (ZAM) distribution. The results of the analysis show that the amplitude of the sideband components is modulated by the low frequency vibration and the modulation amplitude depends on the size of the crack. Based on the obtained results, a new damage index was defined in relation to the strength of the modulation. The new damage index is more sensitive and robust and correlates better with crack size compared to the index based on the amplitude of the sidebands.
\end{abstract}

\section{Introduction}

Structures with inhomogeneities or defects exhibit strong nonlinear vibrational and acoustical effects. In particular, strong nonlinear effects were observed in structures with cracks. These effects include the generation of higher harmonics and intermodulation of a high-frequency acoustic wave by a low-frequency vibration [1] and provide the foundation for developing different techniques for nondestructive testing.

The vibroacoustic modulation (VAM) method is based on the fact that a high-frequency ultrasound probing wave propagating in a structure is modulated by a low-frequency vibration. The modulation is generated by the nonlinear interaction of waves caused by the presence of the crack. The mechanisms, however, behind these effects are still poorly understood [2-4]. The phenomenon of VAM is usually measured in the frequency domain and it is manifested as sidebands around the carrier peak of the ultrasound wave at frequencies equal to the sum and difference of the excitation frequencies and their integer multiples. Modulation effects have been observed in several applications. Ekimov et al. [5] employed VAM of high-frequency torsional waves for crack detection in a rod. Zaitsev et al. [6] presented applications of nonlinear modulation for crack detection in structures and discussed possible sources of nonlinearity in damaged structures. Donskoy and Sutin [7] used VAM to investigate the existence of cracks, delaminations, or poor quality bonding. Further application of VAM techniques can be found in Zagrai et al. [8] who studied crack detection in aluminum plates. Duffour et al. [9] investigated the sensitivity of VAM technique and compared the conventional damping test with an impact-based vibroacoustic modulation. The majority of the existing studies are related to the detection of damage in metallic structures. More recently, studies of the application of VAM techniques to composite structures $[10,11]$ and chiral sandwich panels [12] have been reported.

When VAM is applied for damage detection, damage indices are defined relating the size of the damage to 
the intensity of modulation. These indices rely on the amplitude of the carrier frequency at the sidebands. Despite the successful application of VAM in various damage problems, it appears that the damage indices in the frequency domain used so far are not accurate and in many cases provide unreliable results [13].

The primary aim of the present work is to investigate vibroacoustic modulation in the time-frequency domain by employing time-frequency analysis based on the Zhao-AtlasMarks (ZAM) distribution, which has the advantage of significantly reducing cross-terms between signal components, through its cone-shaped kernel function. We hypothesized that the characteristics of modulation responses in time domain might be proved more sensitive compared to those in the frequency domain and the combination of both could lead to damage indices that are more sensitive and robust. Furthermore, it is believed that the time-frequency analysis of the modulation responses can highlight the underlying nonlinear mechanisms and enable more efficient applications of the method for damage detection.

\section{Methodology}

2.1. Vibroacoustic Modulation (VAM) Technique. In structures with damage (e.g., cracks), strong nonlinear vibrational and acoustical effects occur. Exploitation of these phenomena has led to the formation of the vibroacoustic modulation (VAM) technique, which is probably the most widely used nonlinear, nondestructive testing (NDT) method for crack detection. In particular, the VAM technique involves monitoring of the amplitude modulation of a high-frequency $\left(f_{H}\right)$ vibration field transmitted through a cracked specimen undergoing an additional low-frequency $\left(f_{L}\right)$ structural vibration (typically one of the first structural modes). If the specimen is undamaged and appropriately supported, the two vibration fields do not interact. However if a crack is present, then the low-frequency structural vibration slowly opens and closes the crack. This periodically modifies the dynamic characteristics of the system, hence, modulating the amplitude of the ultrasound transmitted through a cracked specimen. This modulation expresses itself as sidebands $\left(f_{S_{k}}^{ \pm}\right)$ around the high-frequency component $f_{H}$ at frequencies equal to the sum and difference of the excitation frequencies and their integer multiples, that is,

$$
f_{S_{k}}^{ \pm}=f_{H} \pm k f_{L}, \quad k=1,2,3, \ldots
$$

The intensity of the modulation is related to the severity of the damage and has been used so far as a spectral FFT-based damage index $\left(\mathrm{DI}_{\mathrm{FFT}}\right)$ in the form

$$
\operatorname{DI}_{\mathrm{FFT}}=\frac{\left(\left|\operatorname{FFT}\left(f_{S_{1}}^{-}\right)\right|+\left|\operatorname{FFT}\left(f_{S_{1}}^{+}\right)\right|\right)}{2\left|\operatorname{FFT}\left(f_{H}\right)\right|},
$$

where $\left|\operatorname{FFT}\left(f_{S_{1}, H}^{ \pm}\right)\right|$denote the FFT magnitude at the first left and right sidebands and $f_{H}$, respectively.

2.2. Zhao-Atlas-Marks (ZAM) Distribution. Time-frequency (TF) analysis provides the means for exploiting the energyrelated characteristics of the crack response signals that may vary in both time and frequency. Many of the TF approaches suffer from the effect of the appearance of crossterms, which deteriorate the discrimination power at the TF domain. To avoid this distortion, the Zhao-Atlas-Marks (ZAM) distribution [7] was adopted as a methodological tool to express the information in a clearer way at the TF domain. In particular, ZAM distribution belongs to the category of quadratic time-frequency representations and, especially, to the group of reduced interference distributions (RIDs). RIDs are members of Cohen's class and thus, for a time series $X(t)$, they can be described by the following general expression:

$$
\begin{array}{r}
\operatorname{RID}_{X}(t, f ; \Phi)=\iint_{-\infty}^{+\infty} \Phi(\xi, \tau) A_{X}(\xi, \tau) \\
\times e^{-j 2 \pi(f \tau+\xi t)} d \xi d \tau
\end{array}
$$

where $t$ and $f$ denote time and frequency, respectively, while $\tau$ and $\xi$ denote the delay and the doppler, respectively, in the ambiguity plane. $A_{X}(\xi, \tau)$ represents the ambiguity function, which is associated with the Wigner-Ville distribution via a two-dimensional Fourier transform [8]. $\Phi(\xi, \tau)$ is the, so-called, parameterization or kernel function. The ZAM distribution is derived by choosing the kernel function as follows:

$$
\Phi(\xi, \tau)=h(\tau)|\tau| \frac{\sin (\pi \xi \tau)}{\pi \xi \tau}
$$

where $h(\tau)$ is a window function that leads to smoothing along the frequency axis. Thus, the following expression can be obtained that defines the ZAM distribution:

$$
\begin{array}{r}
\operatorname{ZAM}_{X}(t, f)=\int_{-\infty}^{+\infty}\left[h(\tau) \int_{t-|\tau| / 2}^{t+|\tau| / 2} X\left(s+\frac{t}{2}\right)\right. \\
\left.\times X^{*}\left(s-\frac{t}{2}\right) d s\right] \\
\times e^{-j 2 \pi f \tau} d \tau .
\end{array}
$$

ZAM distribution was selected among RIDs due to its advantage of significantly reducing cross-terms between signal components, through its cone-shaped kernel function (4) [7]. In the present study, the ZAM-based TF representation was computed under a $N \times N$ TF resolution; $N$ denotes the number of samples of the signal. Smoothing was performed using Hamming windows of $N / 7$-samples and $N / 6$-samples for time and frequency, respectively.

2.3. ZAM-Based Modulation Effects Analysis. Taking the ZAM distribution of the time series $X(t)$ of beam responses to the VAM stimulation, that is, $\operatorname{ZAM}_{X}(t, f)$, a more detailed exploitation of the modulation effects can be achieved by analyzing the mean amplitude and fluctuation of $\mathrm{ZAM}_{X}(t, f)$ at the main sidebands $\left(f_{S_{k}}^{ \pm}\right)$around the high-frequency component, that is, mean/fluct $\left(\left|\mathrm{ZAM}_{X}\left(t, f_{S_{1}}^{ \pm}\right)\right|\right)$, along with the mean amplitude and fluctuation of $\operatorname{ZAM}_{X}(t, f)$ at the $f_{H}$ excitation frequency, that is, mean/fluct $\left(\left|Z_{X} M_{X}\left(t, f_{H}\right)\right|\right)$. From this perspective, changes in the mean value and 


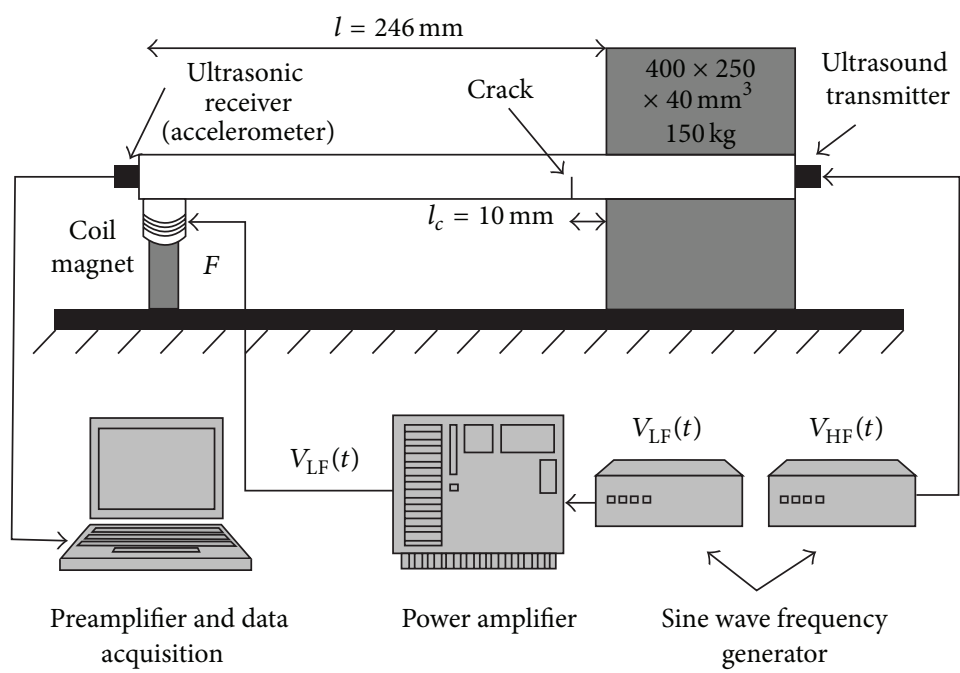

(a)

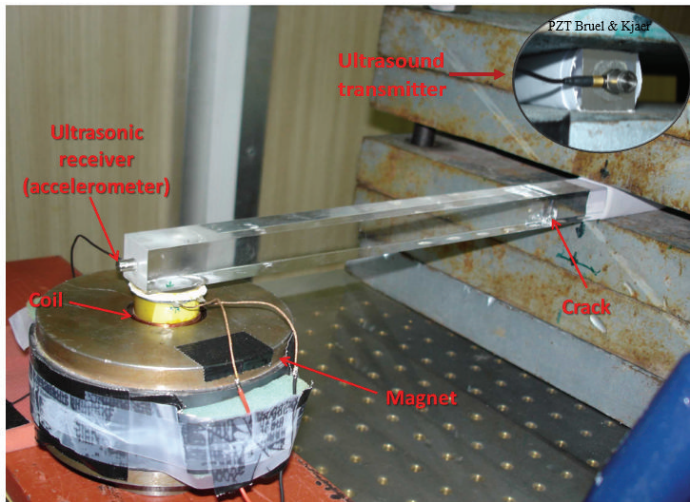

(b)

FIGURE 1: A schematic representation (a) and the actual realization (b) of the experimental setup.

the dynamic range of amplitude fluctuation, combined with the inspection of the spectral characteristics of this fluctuation, could correlate with the crack depth and provide insight into the way the presence of the crack affects the beam response during VAM stimulation.

\section{Experiments}

Tests were performed on Plexiglas beams to obtain nonlinear modulation responses for further signal processing. The beams used in the experiments had dimensions of $2 \times 2 \times$ $40 \mathrm{~cm}$ and they were clamped between two heavy steel jaws. To avoid additional damping and distortion due to couplings, the beam was excited with a force $F$ by using a small voice coil weighting $2 \mathrm{gr}$ attached to the beam. The coil was placed in the field of a permanent magnet and was excited by two waveform generators using sinus signals. A miniature transducer was used to pick up the vibration response, which was transferred to an acquisition system and stored for further analysis. A very narrow cut was initially introduced to the beam. Next, the beam was subjected to controlled dynamical loading which caused crack propagation. Due to the structure of Plexiglas, the propagation of the crack could not be accurately controlled resulting in arbitrary crack depths. A Bruel and Kjaer 4393 piezoelectric charge transducer was used for the high-frequency excitation. A schematic representation and a photo from the actual implementation set-up are depicted in Figures 1(a) and 1(b), respectively. Initially, a fatigue crack of $7 \%$ depth was introduced at $l_{c}=10 \mathrm{~mm}$ from the clamped end. Then, its depth was increased to $20 \%$ and finally to $45 \%$. During the experiments two continuous sine waves were simultaneously introduced to the beam. The first $\left(V_{\mathrm{HF}}(t)\right)$ was the high-frequency ultrasound probe wave at $f_{H}=31.3 \mathrm{kHz}$. The second wave $\left(V_{\mathrm{LF}}(t)\right)$ was the low-frequency vibration at a frequency of $f_{L}=92 \mathrm{~Hz}$, equal to the resonance frequency of the intact beam. The sampling frequency used was $192 \mathrm{kHz}$. Figure 2 depicts an excerpt from the measured response

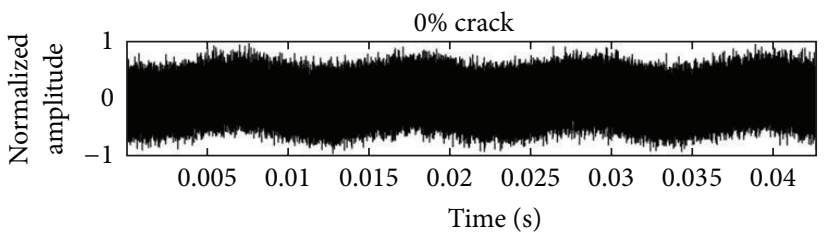

(a)

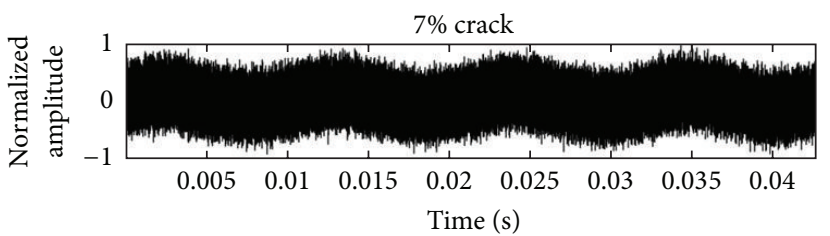

(b)

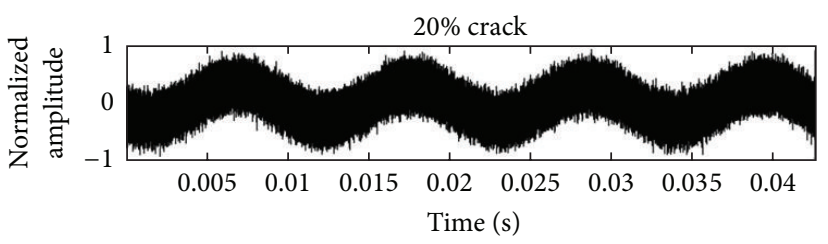

(c)

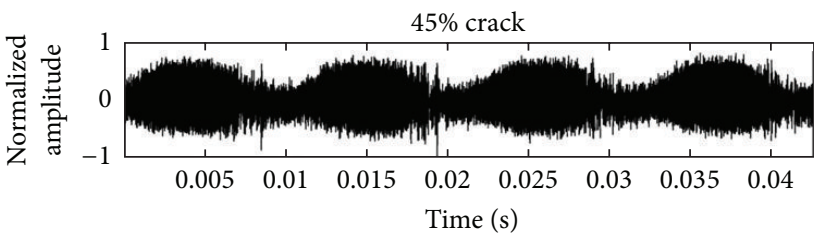

(d)

FIGURE 2: The experimental data excerpt (8192 samples, sampling frequency $192 \mathrm{kHz}$ ) used in the ZAM analysis for the uncracked, 7\%, $20 \%$, and $45 \%$ of the crack depth cases ((a) to (d)). 


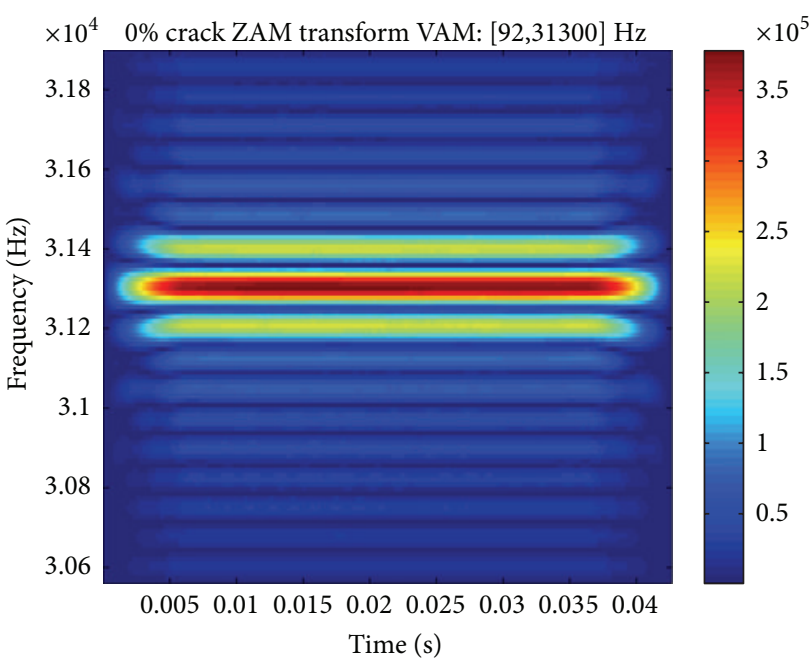

(a)

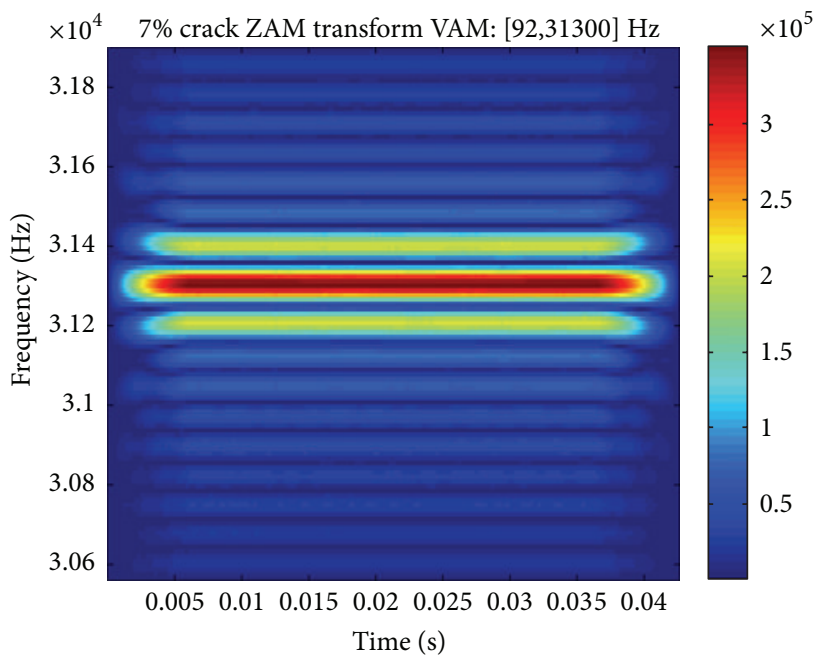

(c)

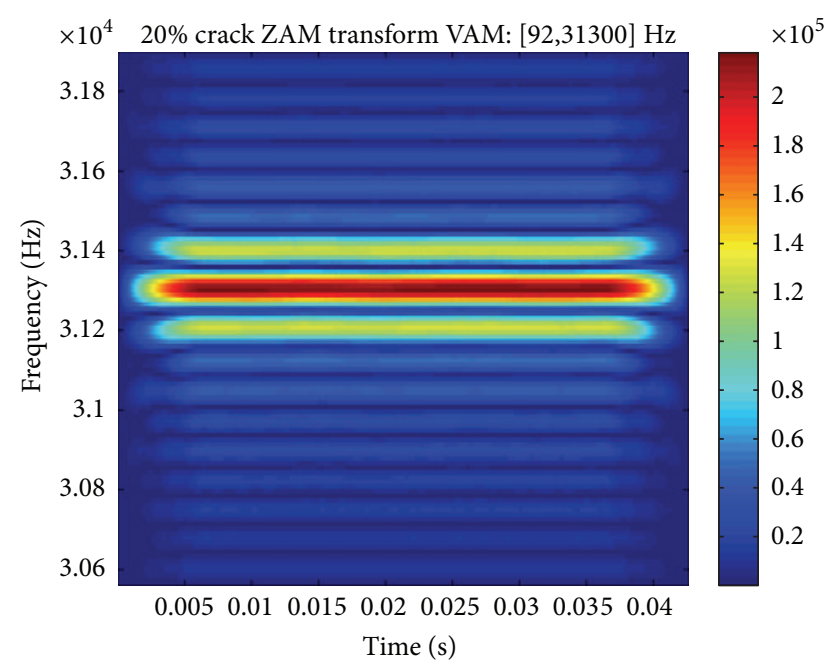

(b)

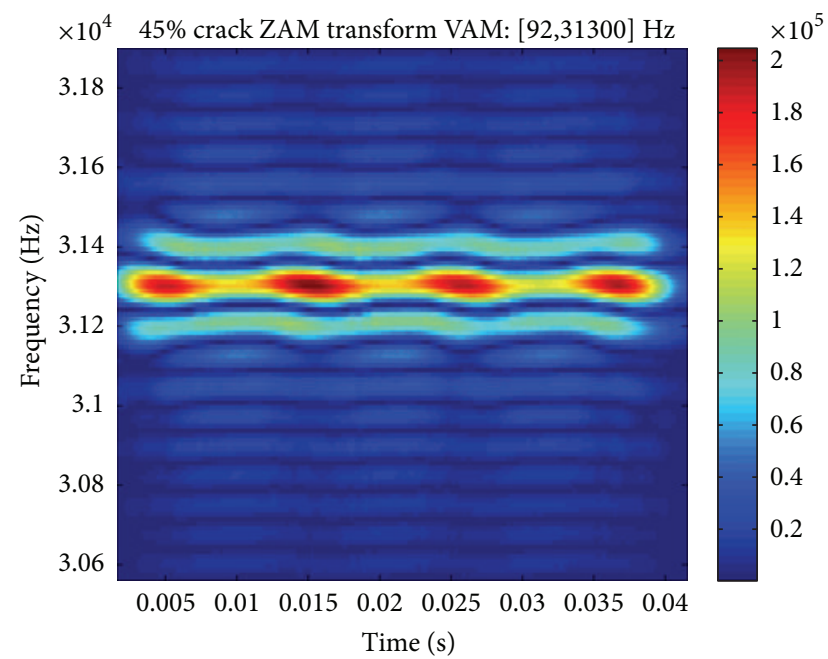

(d)

FIGURE 3: Results from the ZAM analysis of the experimental data of Figure 2 for $0 \%$ (a), 7\% (c), 20\% (b), and 45\% (d) crack size, respectively.

for the uncracked and the three different crack depth cases ((a) to (d)), respectively. As it can be seen from Figure 1, strong modulation components are present and increase with increasing crack depth.

\section{Results and Discussion}

Figure 3 shows the estimated $\operatorname{ZAM}_{X}(t, f)$ of the data depicted in Figure 2 for the four examined crack depths, that is, $0 \%$, $7 \%, 20 \%$, and $45 \%$, zoomed in the area of the $f_{H}=31.3 \mathrm{kHz}$.

Apparently, from these plots it is clear that a series of $f_{S_{k}}^{ \pm}$ sidebands is evident, with the $f_{S_{1}}^{ \pm}$at $f_{S_{1}}^{-}=31208 \mathrm{~Hz}$ and $f_{S_{1}}^{+}=$ $31392 \mathrm{~Hz}$ being the most noticeable, compared to the rest. It is noteworthy that as the crack depth is increased towards $45 \%$ (Figure 3(d)), a fluctuation at the $f_{S_{1}}^{ \pm}$frequencies is noticed, whereas there is a more concentrated activity at $f_{H}$ across the time axis (in the form of peaks rather than frequency line ridges), indicating, possibly, the existence of a "breathingcrack" mechanism. It should be noted that the latter behavior is also noticed in the time domain (see Figure 2), as we move from the $0 \%$ to the $45 \%$ crack depth. More specifically, the periodic behavior of the breathing mechanism is clearly noticed in the modulated amplitude of the time series, with a more profound example being the case of $20 \%$ crack depth (Figure 2(c)), where the $92 \mathrm{~Hz}$ imposed frequency is driving the breathing effect. Nevertheless, when focusing at the highfrequency area, as the subfigures of Figure 3 do, only the case of $45 \%$ crack depth reflects the consequences of the breathing effect at the central and side lobe-frequencies, as previously described (see Figure 3(d)).

The amplitude of the estimated $\operatorname{ZAM}_{X}(t, f)$ of Figure 3 at the corresponding VAM frequencies, that is, $31392 \mathrm{~Hz}$ (a), $31300 \mathrm{~Hz}$ (b), and $31208 \mathrm{~Hz}$ (c) for each crack size (0\%,7\%, $20 \%$, and $45 \%$ ), respectively, is depicted in Figure 4 . From the latter, it is clear that the $\operatorname{ZAM}_{X}(t, f)$ amplitude is inversely 


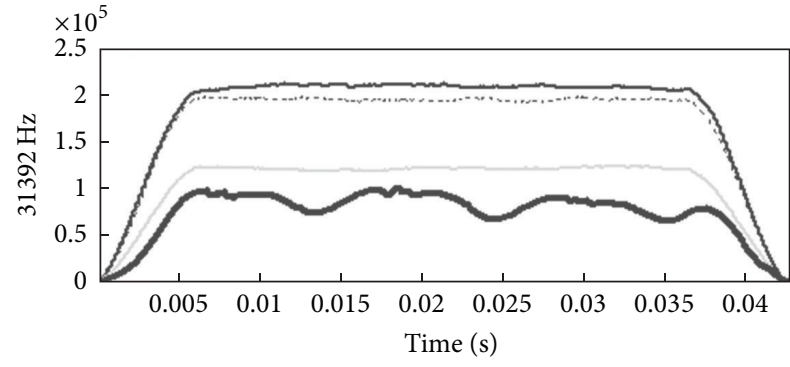

(a)

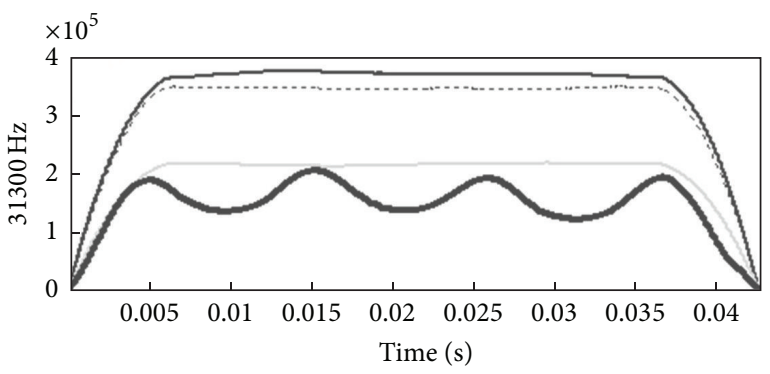

(b)

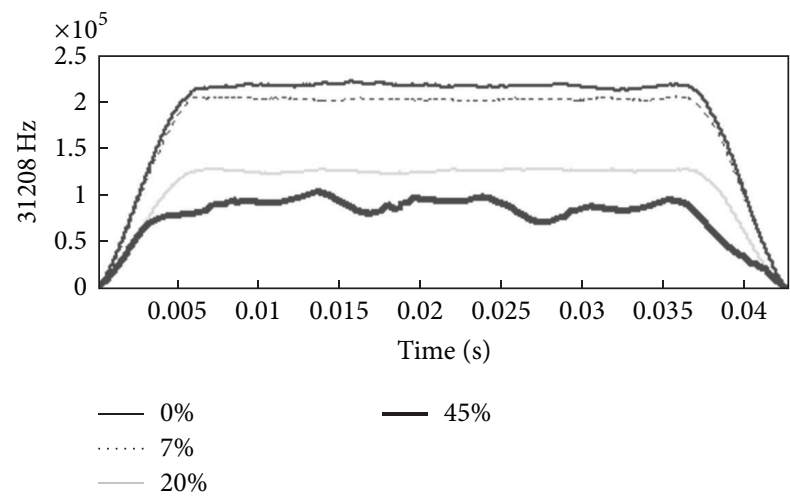

(c)

Figure 4: The amplitude fluctuation of the ZAM transform at the corresponding VAM frequencies, that is, $31392 \mathrm{~Hz}$ (a), $31300 \mathrm{~Hz}$ (b), and $31208 \mathrm{~Hz}$ (c) for each crack size $(0 \%, 7 \%, 20 \%$, and $45 \%)$, respectively.

proportional to the crack depth, whereas the amplitude fluctuation fluct $\left(\left|\operatorname{ZAM}_{X}\left(t, f_{S_{1 H}^{ \pm}}^{ \pm}\right)\right|\right)$is highly increased as the crack depth also increases. This might be justified when taking into account the occurrence of nonlinear dissipation effects due to "crack breathing" that are more pronounced as the crack depth increases. Moreover, there is a clear periodicity in the amplitude modulation for the case of $f_{H}$ (Figure 4(b)); a noticeable, yet not so intense, one is evident in the amplitude modulation of $f_{S_{1}}^{ \pm}$. This is further examined in Figure 5, where the spectrum of the amplitude fluctuation of the ZAM transform at the corresponding VAM frequencies, that is, $31392 \mathrm{~Hz}$ (a), $31300 \mathrm{~Hz}$ (b), and $31208 \mathrm{~Hz}$ (c), for the crack size of $45 \%$, respectively, is illustrated.

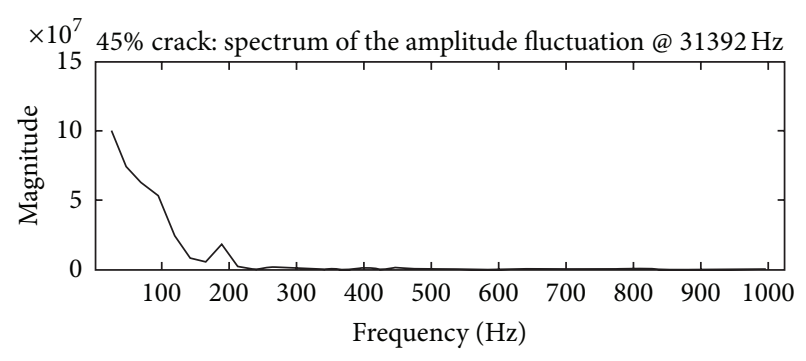

(a)

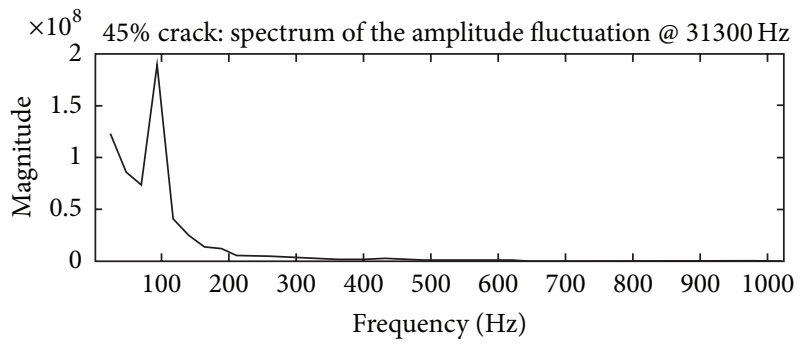

(b)

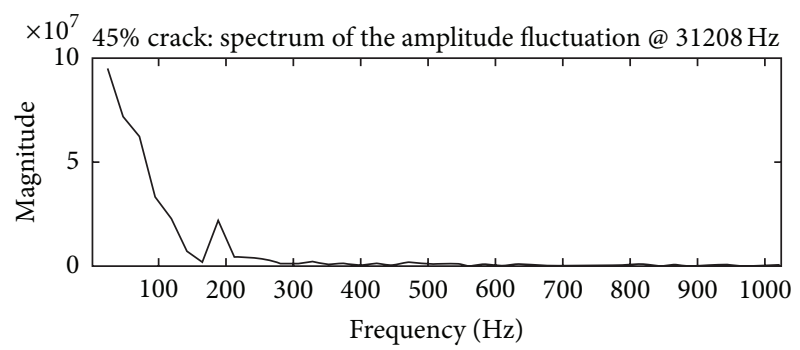

(c)

FIGURE 5: The spectrum of the amplitude fluctuation of the ZAM transform at the corresponding VAM frequencies, that is, $31392 \mathrm{~Hz}$ (a), $31300 \mathrm{~Hz}$ (b), and $31208 \mathrm{~Hz}$ (c), for the crack size of $45 \%$, respectively.

As it is clear from Figure 5, the low excitation frequency $f_{L}=92 \mathrm{~Hz}$ modulates the amplitude of $\mathrm{ZAM}_{X}\left(t, f_{H}\right)$ (Figure 5(b)), whereas mainly the first harmonic of $f_{L}$, that is, $2 f_{L}=184 \mathrm{~Hz}$, causes the amplitude fluctuation $\left|Z_{X} M_{X}\left(t, f_{S_{1}}^{ \pm}\right)\right|$(Figures 5(a) and 5(c), resp.).

Focusing at the $45 \%$ crack depth case, the frequency modulation (fluctuation of ridges) seen in Figure 3(d) is further examined. In particular, Figure 6 reveals the corresponding spectral characteristics of this modulation at the corresponding VAM frequencies, that is, $31392 \mathrm{~Hz}$ (a), $31300 \mathrm{~Hz}$ (b), and $31208 \mathrm{~Hz}$ (c).

Similarly to the spectral characteristics of the amplitude modulation of ZAM transform seen in Figure 5, here the low excitation frequency $f_{L}=92 \mathrm{~Hz}$ modulates the frequency fluctuation $\left|\mathrm{ZAM}_{X}\left(t, f_{S_{1}}^{ \pm}\right)\right|$(Figures 6(a) and 6(c), resp.), whereas a coexistence of $f_{L}=92$ and $2 f_{L}=184 \mathrm{~Hz}$ modulates the frequency fluctuation of $\operatorname{ZAM}_{X}\left(t, f_{H}\right)$ (Figure 6(b)). The separate damage indices (sDI) (all normalized to the corresponding value of $45 \%$ crack depth case after 


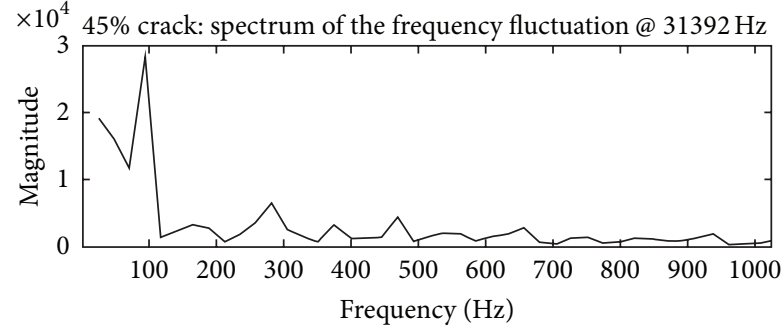

(a)

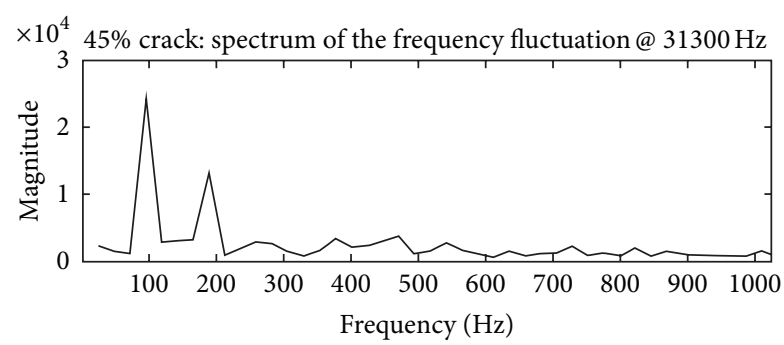

(b)

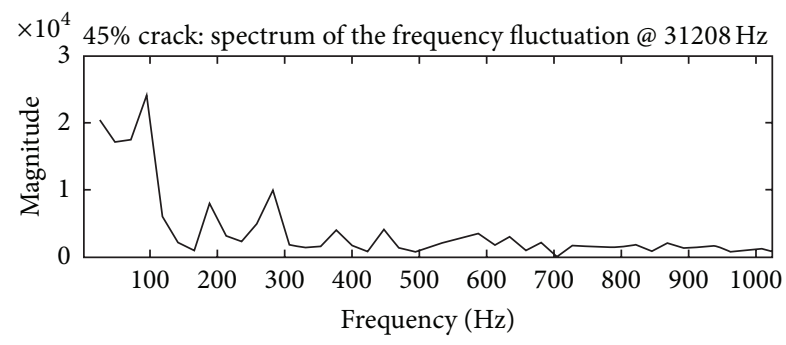

(c)

FIGURE 6: The spectrum of the frequency modulation of the ZAM transform at the corresponding VAM frequencies, that is, $31392 \mathrm{~Hz}$ (a), $31300 \mathrm{~Hz}$ (b), and $31208 \mathrm{~Hz}$ (c), for the crack size of $45 \%$, respectively.

bias elimination for the $0 \%$ crack depth case) based on the $1 /\left|\mathrm{ZAM}_{X}(t, f)\right|$ (first row), the MAX-MIN range (second row), the corresponding standard deviation (third row) of the ZAM transform, and the normalized FFT magnitude (fourth row), at the corresponding VAM frequencies, that is, $31208 \mathrm{~Hz}$ (left column), $31300 \mathrm{~Hz}$ (middle column), and $31392 \mathrm{~Hz}$ (right column), respectively, are shown in Figure 7. From the latter it is deduced that the sensitivity of $1 /\left|Z A_{X}(t, f)\right|$ according to the crack depth change is significantly higher than all other sDI, which mainly capture the transition from $20 \%$ to $45 \%$ crack depth, exhibiting less efficient performance in tracking smaller cracks.

Consequently, the mean value of the sDI for the case of the $1 /\left|Z_{X A}(t, f)\right|$ (Figure 7 , first row) could be defined as the most efficient ZAM-based DI, namely, $\mathrm{DI}_{\mathrm{ZAM}}$. Figure 8 depicts the $\mathrm{DI}_{Z A M}$ along with the $\mathrm{DI}_{\mathrm{FFT}}$ defined in (2). Apparently, the $\mathrm{DI}_{\mathrm{ZAM}}$ surpasses $\mathrm{DI}_{\mathrm{FFT}}$ in terms of higher sensitivity in the crack depth change, as it better captures crack changes, even in small crack depths.
When comparing the presented work with the one of Zaitsev et al. [6], a similar behavior in the crack detection could be identified. Both works conclude that the damage index based on the amplitude modulation is better than the one based on the frequency modulation. Nevertheless, the latter damage index of [6] unexpectedly increases nonmonotonically as the severity of crack increases; here, as it is derived from Figure 7 (second and third rows), the damage indices based on the frequency fluctuation (range and std) of the ZAM distribution increase monotonically with the crack depth, exhibiting, though, reduced sensitivity in the small cracks identification. This, in turn, is compensated by the damage index based on the inverse of the ZAM amplitude. Moreover, the analysis in [6] is prone to the mode-mixing effect; that is, a single intrinsic mode function (IMF) derived from the Empirical Mode Decomposition employed in [6] consists either of signals of widely disparate scales or a signal of a similar scale residing in different IMF components. Mode-mixing is often a consequence of signal intermittency, which could not only cause serious aliasing in the time-frequency distribution, but also make the physical meaning of individual IMF unclear [9]. Perhaps the mode-mixing effect could be the reason for this unexpected behavior of Zaitsev's et al. [6] damage index based on the frequency modulation. The proposed analysis here does not produce any mode-mixing effect, as it is clearly shown in the time-frequency distributions of Figure 3, making the relevant damage indices more robust to any signal intermittencies.

\section{Conclusion}

In this work, the investigation of the vibroacoustic modulation of cracked beam is approached in the time-frequency domain, using time-frequency analysis based on the ZhaoAtlas-Marks (ZAM) distribution. ZAMs efficient timefrequency representation of the vibrational information, with reduced cross-terms between signal components through its cone-shaped kernel function, allowed for detailed monitoring of the VAM effects on beam behavior due to the existence of a crack. The hypothesis adopted here, that is, the characteristics of modulation responses in time domain might be proved more sensitive compared to those in the frequency domain and the combination of both could lead to damage indices that are more sensitive and robust, was proved valid. This was justified by the experimental results derived when applying VAM on Plexiglas beams with a varying crack depth of $0 \%, 7 \%, 20 \%$, and $45 \%$. Considering the responses at the ZAM domain and, especially, the reduction of mean $Z A M$ amplitude at the sidebands and excitation high frequency with the increase of the crack depth a new damage index was formed, that is, $\mathrm{DI}_{\mathrm{ZAM}}$. The latter led to a more sensitive response compared to the one based on the spectral characteristics of the beam response, that is, $\mathrm{DI}_{\mathrm{FFT}}$, better capturing crack changes, even in small crack depths. The promising results presented here enable more efficient applications of the proposed method in nondestructive damage detection applications. 

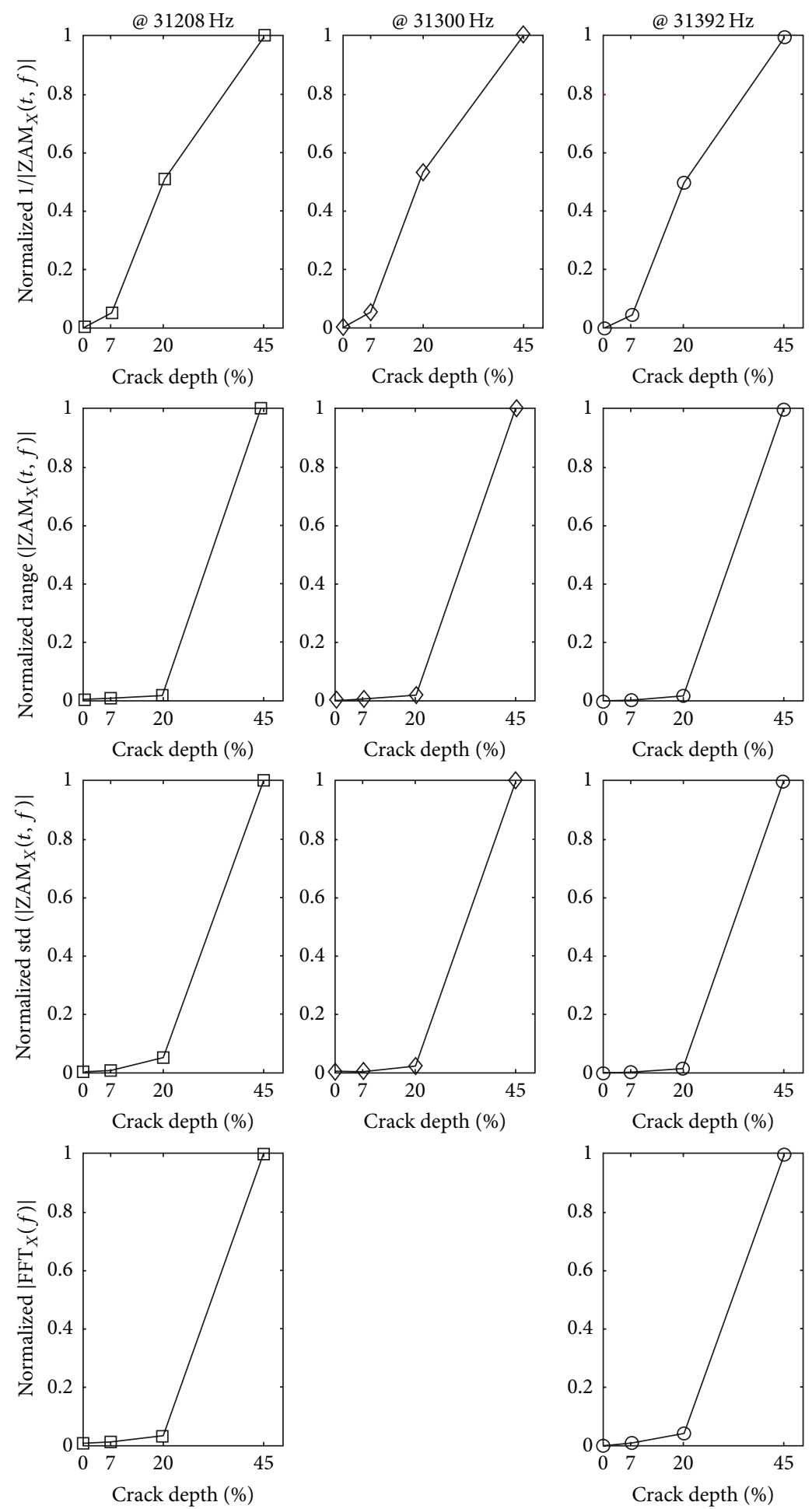

FIGURE 7: The separate damage indices (sDI) based on the $1 /\left|Z_{X} M_{X}(t, f)\right|$ (first row), the MAX-MIN range (second row), the corresponding standard deviation (third row) of the ZAM transform, and the normalized FFT magnitude (fourth row) at the corresponding VAM frequencies, that is, $31208 \mathrm{~Hz}$ (left column), $31300 \mathrm{~Hz}$ (middle column), and $31392 \mathrm{~Hz}$ (right column), respectively. Note that, for the FFTbased analysis, only the $31208 \mathrm{~Hz}$ (left column) and $31392 \mathrm{~Hz}$ (right column) were considered, since the FFT amplitude at the central highfrequency $(31300 \mathrm{~Hz})$ was used as a normalization factor. Moreover, in the ZAM-based analysis, all values were estimated for the time span of $0.006-0.036 \mathrm{sec}$ to avoid edge effects, while all data samples acquired (92001) were used in the FFT-based analysis to increase its frequency resolution. 


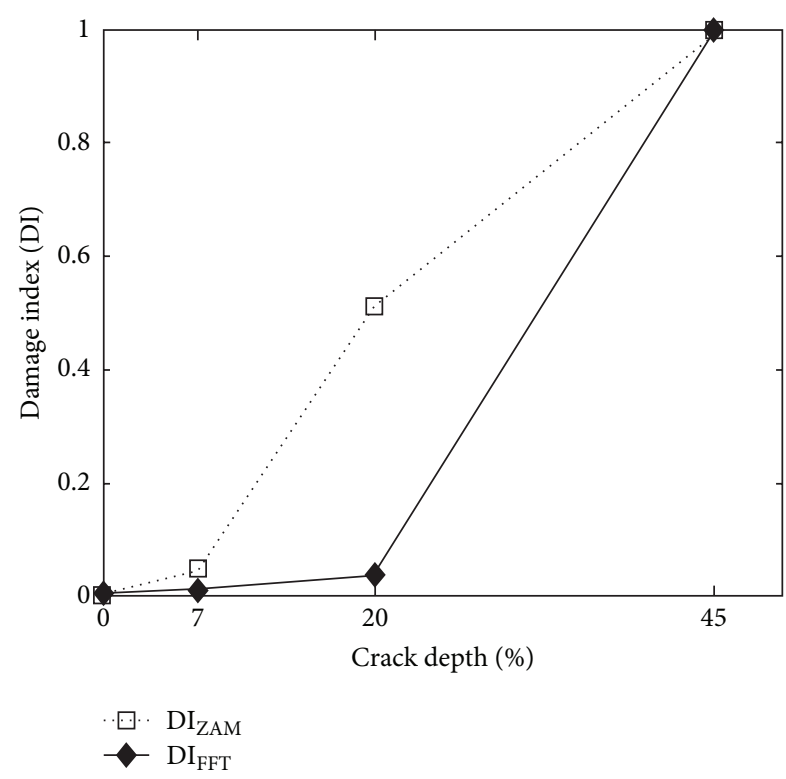

FIgure 8: The damage index (DI) derived as the mean value of the sDI for the case of the $1 /\left|Z_{X A M}(t, f)\right|$ (Figure 7 , first row)-DI ${ }_{\text {ZAM }}$

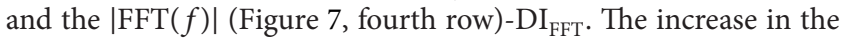
sensitivity of the $\mathrm{DI}_{\mathrm{ZAM}}$ over the $\mathrm{DI}_{\mathrm{FFT}}$ is evident.

\section{Conflict of Interests}

The authors declare that there is no conflict of interests regarding the publication of this paper.

\section{References}

[1] L. A. Ostrovsky and P. Johnson, "Dynamic nonlinear elasticity in geomaterials," Rivista del Nuovo Cimento, vol. 24, no. 7, pp. $1-46,2001$.

[2] A. Moussatov, V. Gusev, and B. Castagnede, "Self-induced hysteresis for nonlinear acoustic waves in cracked material," Physical Review Letters, vol. 90, no. 12, article 124301, 2003.

[3] A. Klepka, W. J. Staszewski, R. B. Jenal, M. Szwedo, J. Iwaniec, and T. Uhl, "Nonlinear acoustics for fatigue crack detectionexperimental investigations of vibro-acoustic wave modulations," Structural Health Monitoring, vol. 11, no. 2, pp. 197-211, 2012.

[4] V. Zaitsev, V. Gusev, and B. Castagnede, "Thermoelastic mechanism for logarithmic slow dynamics and memory in elastic wave interactions with individual cracks," Physical Review Letters, vol. 90, no. 7, Article ID 075501, 2003.

[5] A. E. Ekimov, I. N. Didenkulov, and V. V. Kazakov, "Modulation of torsional waves in a rod with a crack," Journal of the Acoustical Society of America, vol. 106, no. 3, pp. 1289-1292, 1999.

[6] V. Zaitsev, V. Nazarov, V. Gusev, and B. Castagnede, "Novel nonlinear-modulation acoustic technique for crack detection," NDT \& E International, vol. 39, no. 3, pp. 184-194, 2006.

[7] D. M. Donskoy and A. M. Sutin, "Vibro-acoustic modulation nondestructive evaluation technique," Journal of Intelligent Material Systems and Structures, vol. 9, no. 9, pp. 765-771, 1999.

[8] A. Zagrai, D. Donskoy, A. Chudnovsky, and E. Golovin, "Microand macroscale damage detection using the nonlinear acoustic vibro-modulation technique," Research in Nondestructive Evaluation, vol. 19, no. 2, pp. 104-128, 2008.

[9] P. Duffour, M. Morbidini, and P. Cawley, "Comparison between a type of vibro-acoustic modulation and damping measurement as NDT techniques," NDT \& E International, vol. 39, no. 2, pp. 123-131, 2006.

[10] A. Klepka, L. Pieczonka, W. J. Staszewski, and F. Aymerich, "Impact damage detection in laminated composites by nonlinear vibro-acoustic wave modulation," Composites B: Engineering, 2013.

[11] F. Aymerich and W. J. Staszewski, "Experimental study of impact-damage detection in composite laminates using a crossmodulation vibro-acoustic technique," Structural Health Monitoring, vol. 9, no. 6, pp. 541-553, 2010.

[12] A. Klepka, W. J. Staszewski, D. di Maio, and F. Scarpa, "Impact damage detection in composite chiral sandwich panels using nonlinear vibro-acoustic modulations," Smart Materials and Structures, vol. 22, no. 8, Article ID 084011, 2013.

[13] H. F. Hu, W. J. Staszewski, N. Q. Hu, R. B. Jenal, and G. J. Qin, "Crack detection using nonlinear acoustics and piezoceramic transducers-instantaneous amplitude and frequency analysis," Smart Materials and Structures, vol. 19, no. 6, Article ID 065017, 10 pages, 2010. 

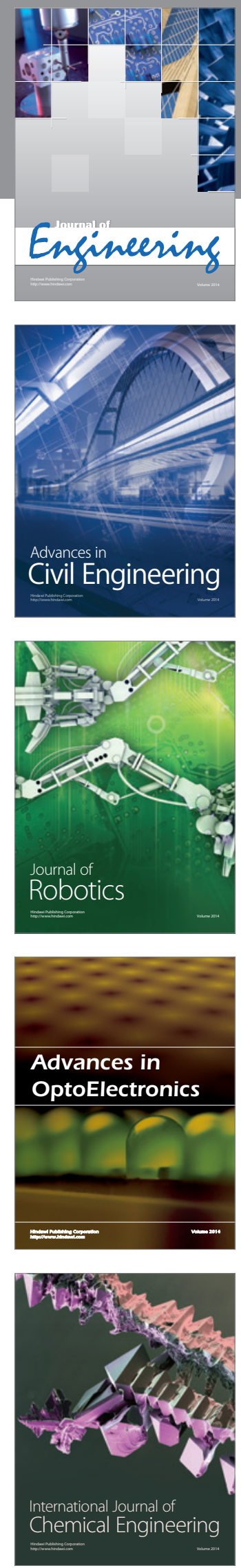

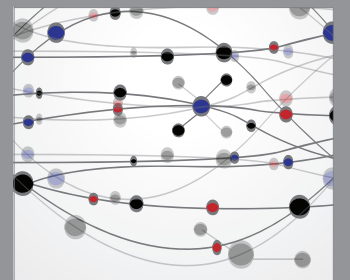

The Scientific World Journal
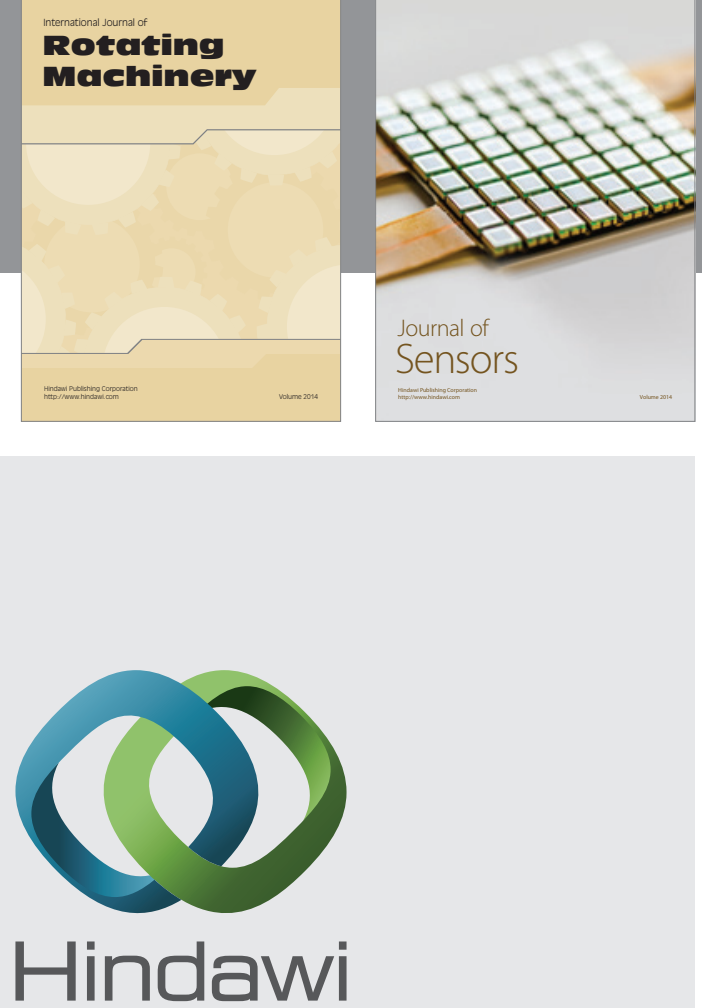

Submit your manuscripts at http://www.hindawi.com
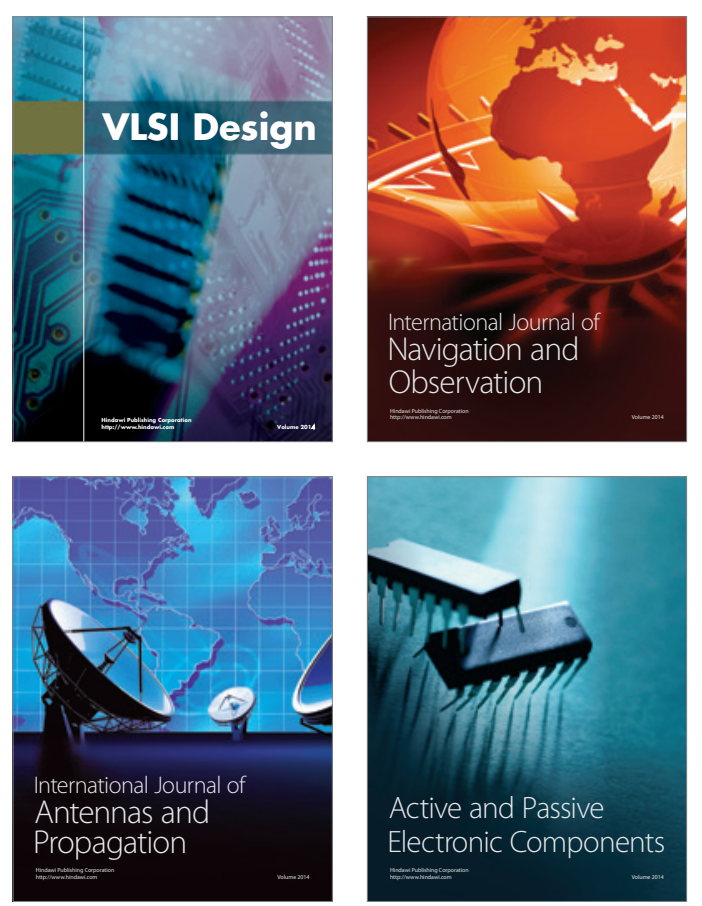
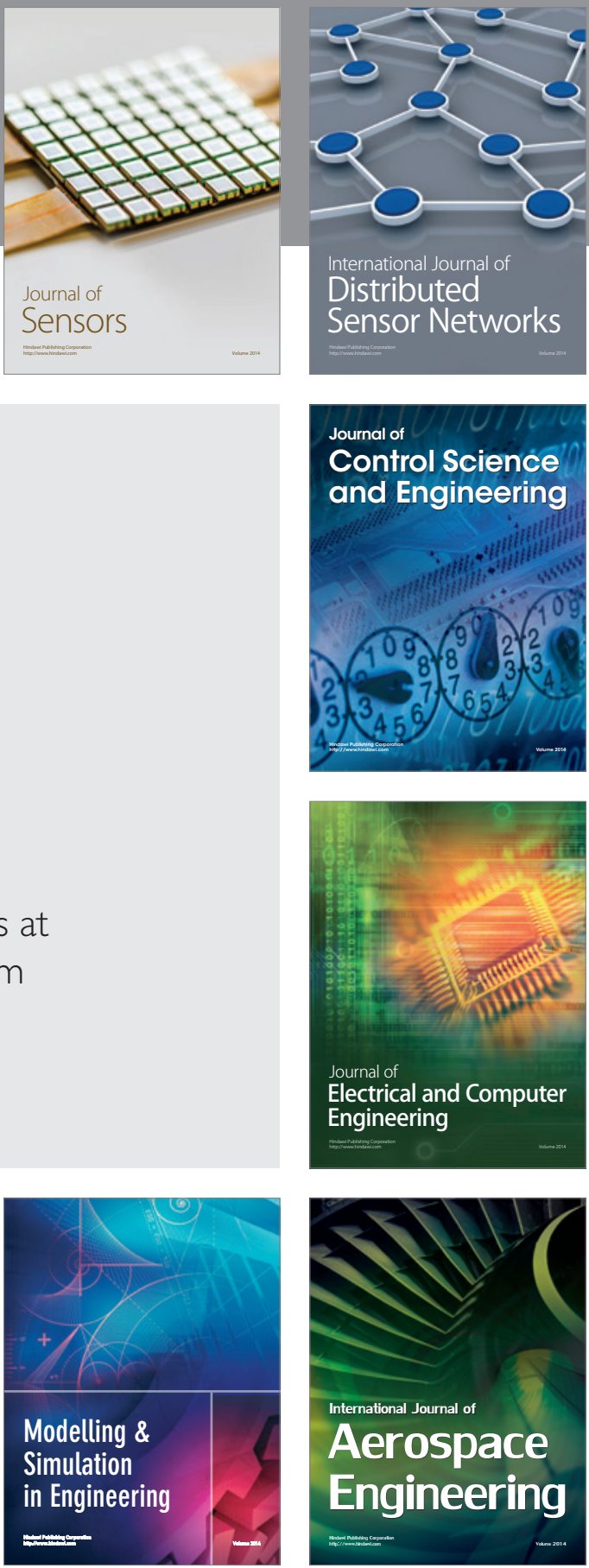

Journal of

Control Science

and Engineering
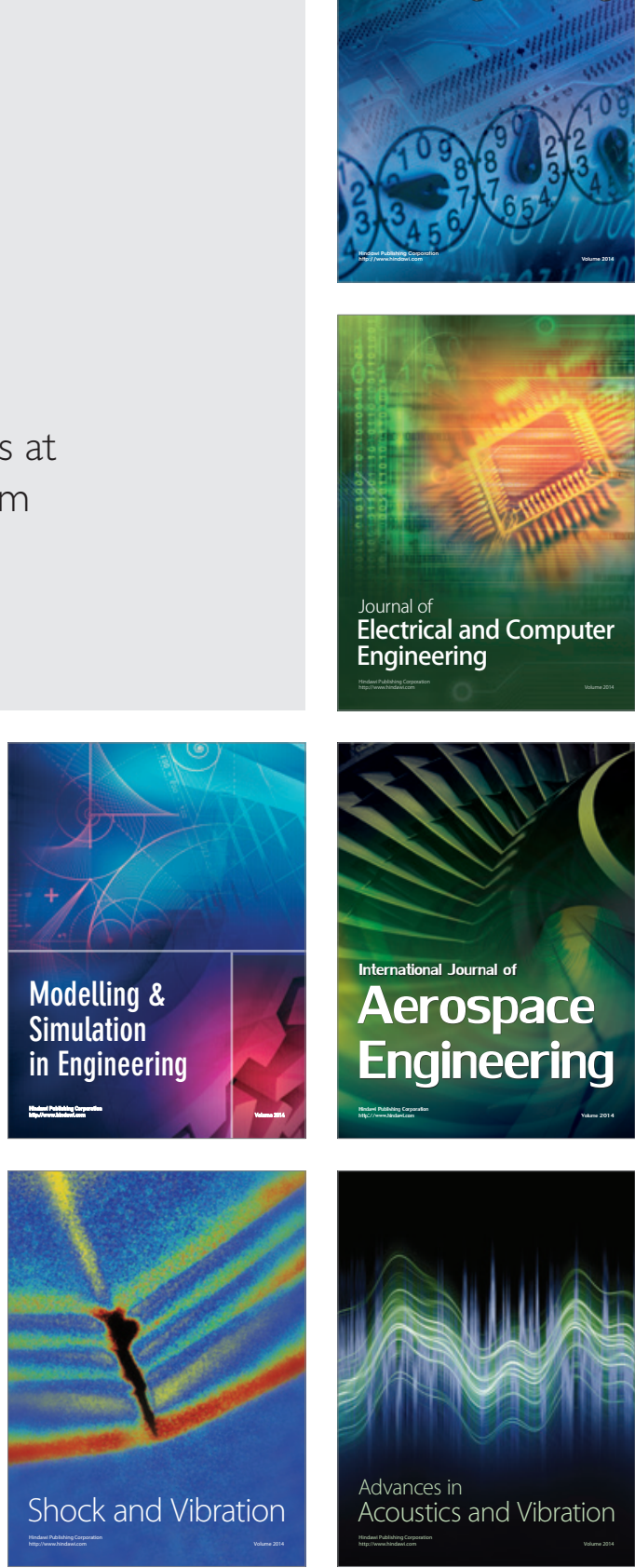